\title{
Plasmon and coupled plasmon-phonon modes in graphene in the presence of a driving electric field
}

\author{
C. X. Zhao, ${ }^{1}$ W. Xu, ${ }^{1,2, *}$ H. M. Dong, ${ }^{3}$ and F. M. Peeters ${ }^{4}$ \\ ${ }^{1}$ Key Laboratory of Materials Physics, Institute of Solid State Physics, Chinese Academy of Sciences, Hefei 230031, China \\ ${ }^{2}$ Department of Physics, Yunnan University, Kunming 650091, China \\ ${ }^{3}$ Department of Physics, China University of Mining and Technology, Xuzhou 221116, China \\ ${ }^{4}$ Department of Physics, University of Antwerp, Groenenborgerlaan 171, B-2020 Antwerpen, Belgium
}

(Received 8 April 2014; revised manuscript received 7 May 2014; published 29 May 2014)

\begin{abstract}
We present a theoretical study of the plasmon and coupled plasmon-phonon modes induced by intraband electron-electron interaction in graphene in the presence of driving dc electric field. We find that the electric field dependence of these collective excitation modes in graphene differs significantly from that in a conventional two-dimensional electron gas with a parabolic energy spectrum. This is due mainly to the fact that graphene has a linear energy spectrum and the Fermi velocity of electrons in graphene is much larger than the drift velocity of electrons. The obtained results demonstrate that the plasmon and coupled plasmon-phonon modes in graphene can be tuned by applying not only the gate voltage but also the source-to-drain field. The manipulation of plasmon and coupled plasmon-phonon modes by source-to-drain voltage can let graphene be more conveniently applied as an advanced plasmonic material.
\end{abstract}

DOI: 10.1103/PhysRevB.89.195447

PACS number(s): 73.20.Mf, 73.23.-b

\section{INTRODUCTION}

The plasmon is a kind of collective oscillation of electrons in an electron gas system. Its interactions with a light field enable us to manipulate the electromagnetic energy at subwavelength scales [1]. Based on unique features of plasmon oscillations, plasmonics has become an important and fast-growing field of scientific research in recent years. Plasmonics is making an impact on many technological areas including terahertz technology [2], light harvesting [3], quantum information processing [4], and invisibility cloaks [5], to mention but a few. It is known that the noble metals, which are regarded as excellent plasmonic materials [6], are hardly tunable and exhibit large Ohmic losses that limit their applications in optical processing devices. In 2009, Jablan et al. [7] demonstrated that the plasmon in graphene can have very low Ohmic losses in infrared bandwidth and, therefore, proposed that such a feature can be utilized for nanophotonic device applications. Many experimental [8-11] and theoretical $[12,13]$ findings have shown that the basic features of plasmon in graphene differ significantly from those in conventional two-dimensional electron gas (2DEG) systems, such as much stronger field confinement, higher wave velocity, longer propagation length, better tunability via electrostatic gating, etc. These interesting and important features of plasmon in graphene have allowed us to consider that graphene is also an excellent plasmonic material. It can be applied for some areas of plasmonic technology for electro-optical processing that have so far been difficult to achieve with conventional metallic plasmonic materials $[14,15]$.

It has been realized that the plasmon modes in graphene on a dielectric wafer can be efficiently tuned by, e.g., simply applying a gate voltage. This can alter the electron density in graphene and, thus, change the corresponding plasmon frequency. As we know, the plasmon modes in conventional 2DEG systems depend sensitively on a driving dc electric field

\footnotetext{
*wenxu_issp@aliyun.com
}

applied along the 2D plane of the 2DEG [16-18]. In such a case, a Doppler-type of shift in plasmon frequency can be observed. The application of a driving electric field can lead to the generation of plasmon emission. Therefore, it is necessary and significant to examine the plasmon modes in graphene driven by a dc electric field. Generally, when a driving electric field is applied to an electron gas, the electrons are not in equilibrium states anymore. The nonequilibrium distribution of electrons has to be taken into consideration in order to study the physical properties induced by electron-electron interaction. As a result, the plasmon modes of an electron gas can be affected strongly by the driving electric field. Because an electron in graphene is with a linear energy spectrum, it is expected that the dependence of plasmon modes in graphene on a driving electric field can differ greatly from that in a conventional 2DEG. Moreover, the plasmon frequency in graphene ranges from terahertz to infrared bandwidth $[12,19]$ and the optical phonon energy in it is about 196 mev. Thus, the plasmon can strongly couple with the optic-phonon modes in graphene. Hence, it is worth also to look into how the driving dc electric filed can affect the coupled plasmon-phonon modes in graphene. In this article, we present a detailed theoretical investigation on the plasmon and coupled plasmonphonon modes in graphene driven by dc electric field at room temperature.

\section{THEORETICAL APPROACH}

Our previous theoretical study [19] has shown that the collective excitations of electrons in graphene can be achieved via intraband and interband electronic transition channels. The intraband plasmon and coupled plasmon-phonon excitations are free of Landau damping, whereas the interband collective excitations are damped by the Landau damping effect due to single-particle excitation. Therefore, in the present study we investigate the plasmon and the coupled plasmon-phonon modes via intraband transition channel in graphene. We consider a graphene sheet on top of a dielectric wafer such as $\mathrm{SiO}_{2}$ or $\mathrm{Si}$. The conducting carriers in graphene are electrons in 
the presence of a positive gate voltage and the electron density in graphene can be tuned by the applied gated voltage $V_{g}$. In the presence of an in-plane source-to-drain electric voltage $V_{s d}$ (taken along the $x$ direction of the graphene sheet), the driving electric field is $F_{x}=V_{s d} / L$ with $L$ being the length for sourceto-drain electrodes. The wave function and energy spectrum for an electron in graphene are, respectively, $E(\mathbf{k})=\gamma|\mathbf{k}|$ and $\psi_{\mathbf{k}}(\mathbf{r})=|\mathbf{k}\rangle=2^{-1 / 2}\left[1, e^{i \phi}\right] e^{i \mathbf{k} \cdot \mathbf{r}}$. Here $\mathbf{r}=(x, y), \mathbf{k}=\left(k_{x}, k_{y}\right)$ is the wave vector for an electron along the graphene sheet, $\gamma=\hbar v_{F}$ with $v_{F}=10^{8} \mathrm{~cm} / \mathrm{s}$ being the Fermi velocity, and $\phi$ is the angle between $\mathbf{k}$ and the $x$ direction. Under the standard random phase approximation (RPA), the dielectric function in the absence of phonon scattering is given as [19]

$$
\varepsilon_{e e}(\mathbf{q}, \omega)=1-\frac{V_{q}}{2} \sum_{\mathbf{k}}\left(1+A_{\mathbf{k q}}\right) \Pi(\mathbf{k}, \mathbf{q}, \omega),
$$

where $V_{q}=2 \pi e^{2} / \kappa q$ is the 2D Fourier transform of the Coulomb potential, $\kappa$ is the high-frequency dielectric constant of graphene, $\mathbf{q}=\left(q_{x}, q_{y}\right)$ is the momentum change during an electron-electron scattering event, $A_{\mathbf{k q}}=(k+q \cos \theta) / \mid \mathbf{k}+$ $\mathbf{q} \mid$, and $\theta$ is the angle between $\mathbf{k}$ and $\mathbf{q}$. In the presence of optic-phonon scattering, the dielectric function can be obtained by using the self-consistent field theory [19], which results in

$$
\begin{aligned}
\varepsilon_{e p}(\mathbf{q}, \Omega)= & 1-\frac{V_{q}}{2} \sum_{\mathbf{k}}\left(1+A_{\mathbf{k q}}\right) \Pi(\mathbf{k}, \mathbf{q}, \Omega) \\
& -D\left(\omega_{q}, \Omega\right) \sum_{\mathbf{k}}|U(\mathbf{k}, \mathbf{q})|^{2} \Pi(\mathbf{k}, \mathbf{q}, \Omega),
\end{aligned}
$$

where $\mathbf{q}$ is also the phonon wave vector due to momentum conservation law for electron-phonon coupling, $|U(\mathbf{k}, \mathbf{q})|^{2}$ is the square of the electron-phonon interaction matrix element, and $D\left(\omega_{q}, \Omega\right)=2 \hbar \omega_{q} /\left[(\hbar \Omega)^{2}-\left(\hbar \omega_{q}\right)^{2}\right]$ is the phonon propagator with $\hbar \omega_{q}$ being the phonon energy. For graphene, the optic-phonon energy is $\hbar \omega_{q}=196 \mathrm{meV}$ and $|U(\mathbf{k}, \mathbf{q})|^{2} \simeq$ $g^{2} \gamma^{2}$ for electron coupling with optic phonons on the basis of a valence-force-field model [20]. Here, $g=\hbar\left(B / b^{2}\right) / \sqrt{2 \rho \hbar \omega_{q}}$ with $\rho \simeq 6.5 \times 10^{-8} \mathrm{~g} / \mathrm{cm}^{2}$ being the areal density of the graphene sheet, $B \simeq 2$ is a dimensionless parameter, and $b=a / \sqrt{3}$ is the equilibrium bond length with $a=1.42 \AA$. Furthermore, the density-density correlation function (or pair bubble) is given as

$$
\Pi(\mathbf{k}, \mathbf{q}, \omega)=g_{s} g_{v} \frac{\mathcal{F}[E(\mathbf{k}+\mathbf{q})]-\mathcal{F}[E(\mathbf{k})]}{\hbar \omega+E(\mathbf{k}+\mathbf{q})-E(\mathbf{k})+i \delta},
$$

with $\mathcal{F}(E)$ being the energy distribution function for electrons and $g_{s}=2$ and $g_{v}=2$ counting, respectively, the spin and valley degeneracy.

In the presence of a driving dc electric field, the electrons in graphene are accelerated and heated. As a result, they are not in the equilibrium states anymore. The often-used equilibrium distribution functions, such as the Fermi-Dirac function and Maxwell function, cannot be employed to describe accurately the electron distribution in the system. Generally speaking, the nonequilibrium electron distribution function can be obtained by, e.g., solving the corresponding Boltzmann equation or the Monte Carlo method [21]. From a practical point of view, one often takes the modified equilibrium distribution function to describe the nonequilibrium distribution of electrons in an electron gas. In particular, one can assume [22,23] the nonequilibrium energy distribution for electrons in graphene to be as $\mathcal{F}[E(\mathbf{k})] \simeq f\left[E\left(\mathbf{k}^{*}\right)\right]$, where $\mathbf{k}^{*}=\left(k_{x}-k_{F} v_{x} / v_{F}, k_{y}\right)$ is the momentum drifted by electron velocity $v_{x}, k_{F}=\sqrt{\pi n_{e}}$ is the Fermi wave vector with $n_{e}$ being the electron density, and $f(E)=\left[e^{(E-\mu) / k_{B} T_{e}}+1\right]^{-1}$ is the modified Fermi-Dirac distribution function with $T_{e}$ being the electron temperature and $\mu$ the Fermi energy (or chemical potential). By applying the balance equation approach on the basis of the Boltzmann equation, $v_{x}$ and $T_{e}$ can be determined by solving selfconsistently the momentum- and energy-balance equations in which electronic scattering mechanisms are included [22].

In the absence of phonon scattering, the plasmon mode is determined by $\operatorname{Re} \varepsilon_{e e}(q, \omega) \rightarrow 0$. At a long-wavelength $(q \rightarrow$ 0 ) limit, the plasmon frequency for graphene driven by an electric field is obtained as

$$
\omega^{2}=\omega_{p}^{2} C(\varphi)
$$

where $\omega_{p}=\left(2 e^{2} E_{F} q / \hbar^{2} \kappa\right)^{1 / 2}$ is the plasmon frequency in the absence of the driving dc electric field at low-temperature limit $T \rightarrow 0, E_{F}=\gamma k_{F}$ is the Fermi energy in graphene at $T \rightarrow 0$, $\varphi$ is the angle between the plasmon wave vector $\mathbf{q}$ and the $x$ axis, and

$$
\begin{aligned}
C(\varphi)= & \frac{1}{\pi} \int_{0}^{\infty} \int_{0}^{\pi} d \phi d x F(x, \phi) \\
& \times\left[\sin ^{2}(\phi-\varphi)+\sin ^{2}(\phi+\varphi)\right] .
\end{aligned}
$$

Here $\quad F(x, \phi)=\left(e^{\left(\alpha E_{F}-\mu\right) / k_{B} T_{e}}+1\right)^{-1} \quad$ with $\quad \alpha=$ $\sqrt{x^{2}+\left(v_{x} / v_{F}\right)^{2}-2 x\left(v_{x} / v_{F}\right) \cos \phi}$. When the driving electric field $F_{x}$ is absent, the plasmon frequency in graphene shows no angular dependence. As $F_{x}$ is applied, the electron drift velocity $v_{x}$ is generated. Thus, the symmetry of the sample geometry is broken and the plasmon frequency has an angular dependence.

In the presence of phonon coupling, the coupled plasmonphonon mode is determined by $\operatorname{Re} \varepsilon_{e p}(q, \Omega) \rightarrow 0$. At a longwavelength limit, we obtain two excitation modes as

$$
\Omega_{ \pm}=\frac{1}{\sqrt{2}}\left[\left(\omega_{q}^{2}+\omega^{2}\right) \pm \sqrt{\left(\omega_{q}^{2}-\omega^{2}\right)^{2}+4 \omega_{Q}^{4}}\right]^{\frac{1}{2}},
$$

where $\omega_{Q}^{4}=2 \omega_{q} g^{2} \gamma^{2} q^{2} E_{F} C(\varphi) / \hbar^{3} \pi$ and $\omega$ is the plasmon frequency for graphene given by Eq. (4). In the presence of a driving electric field, the coupled plasmon-phonon modes also show an angular dependence.

\section{FURTHER CONSIDERATIONS}

In the present study, we consider a vacuum/graphene/ $\mathrm{SiO}_{2}$ device, which was used for the measurement of high-field transport properties of graphene [24]. In such a device, the major scattering centers to limit electron mobility in graphene are the charged impurities, the acoustic phonons via deformation potential coupling, and the optic phonons via polar optic coupling. By including these electronic scattering mechanisms within the balance-equation approach on the basis of the Boltzmann equation, we have calculated the drift electron velocity $v_{x}$ and electron temperature $T_{e}$ in graphene at a given electric field strength $F_{x}$ via solving self-consistently the momentum- and energy-balance equations [22]. Thus, we 
have obtained the corresponding $I-V_{s d}$ characteristics for different electron densities (or gate voltages) and achieved good agreement with the experimental findings both qualitatively and quantitatively. It should be noted that the presence of the dielectric $\mathrm{SiO}_{2}$ layer in a vacuum/graphene/ $/ \mathrm{SiO}_{2}$ system can affect the plasmon modes in graphene mainly for two reasons. First, because of the mismatch of the dielectric constants at interface of the structure, the well-known mirror image effect is present. Hence, the effective dielectric constant in graphene has to be evaluated from the bare dielectric constants for vacuum, graphene, and the $\mathrm{SiO}_{2}$ wafer using the mirror image method via $[12,25,26]: \kappa=\left(1+\kappa_{\mathrm{SiO}_{2}}\right) / 2 \simeq 2.5$. We note that one often takes $\kappa=1$ for suspended graphene $[19,26]$. This is the main reason why the plasmon frequency in a vacuum/graphene/dielectric-wafer device is lower than that in freestanding graphene. A more detailed and comprehensive study on the effect of the dielectric wafer layer on plasmon modes in graphene was reported recently [27]. Second, in a vacuum/graphene/ $\mathrm{SiO}_{2}$ device the charged impurities are mainly located in the $\mathrm{SiO}_{2}$ layer [22,28]. The charged impurity scattering is the major scattering mechanism to limit electron mobility (or velocity) in graphene at low temperatures $T<$ $20 \mathrm{~K}[22,28]$. Therefore, the charged impurities in $\mathrm{SiO}_{2}$ layer can affect the plasmon frequency at relatively low temperatures. In fact, the impurity scattering can enhance the screening of electrons and lead to the softening of the plasmon frequency. This is a typical result for one- and two-dimensional electronic systems where the scattering by the impurities strongly affects the plasmon dispersion [29-31].

\section{RESULTS AND DISCUSSIONS}

In the previous work [32] we have shown the dependence of drift electron velocity $v_{x}$ and electron temperature $T_{e}$ on $F_{x}$ by solving self-consistently the momentum- and energybalance equations in which the electron interactions with charged impurities, acoustic phonons, and optic phonons are considered. The obtained results agree both qualitatively and quantitatively with those from experimental measurements [24]. In this study, we take the same theoretical approach as documented in Ref. [22] to calculate $v_{x}$ and $T_{e}$ at a given $F_{x}$ and, from them, to calculate the frequencies of the plasmon and coupled plasmon-phonon modes in graphene in the presence of $F_{x}$. All results presented in this parer are obtained for room temperature $T=300 \mathrm{~K}$.

Figure 1 shows the angular dependence of plasmon frequency in graphene for different driving electric fields at a fixed electron density and $q=k_{F}$. We notice the following features. (i) The plasmon frequency depends very little on the angle $\varphi$ for a relatively small driving electric field, e.g., at $F_{x}=2 \mathrm{kV} / \mathrm{cm}$. The stronger dependence of the plasmon frequency on $\varphi$ can be observed at a larger electric field, e.g., at $F_{x}=15 \mathrm{kV} / \mathrm{cm}$. (ii) The maximum plasmon frequency appears at $\varphi=90^{\circ}$. (iii) The plasmon frequency decreases monotonously with increasing driving electric field $F_{x}$ over all $\varphi$ angles. However, the plasmon frequency in graphene shows a much weaker dependence on angle $\varphi$ than that in a conventional 2DEG. It is known that in a conventional 2DEG driven by a dc electric field, the plasmon frequency shows a Doppler-type of shift corresponding to $[16,17]$ :

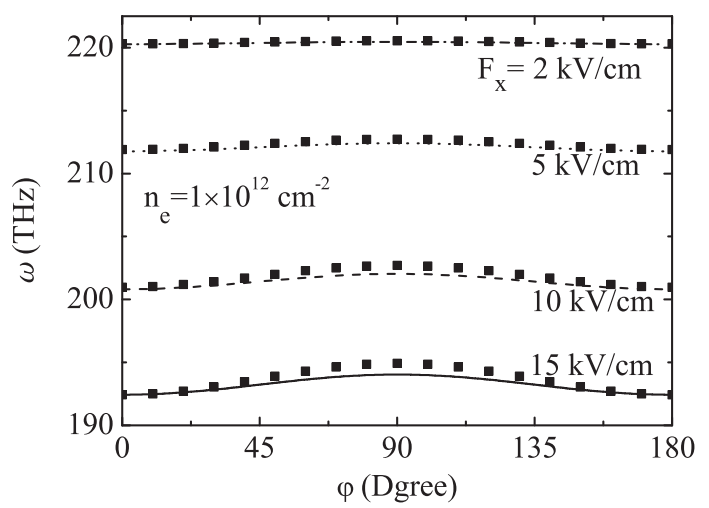

FIG. 1. The angular dependence of plasmon frequency in graphene at the fixed electron density and $q=k_{F} \simeq 1.77 \times$ $10^{6} \mathrm{~cm}^{-1}$ for different driving electric fields. For $F_{x}=2,5,10$, and $15 \mathrm{kV} / \mathrm{cm}$, the corresponding drift velocities and electron temperatures are, respectively, $5.9 \times 10^{6}, 10.6 \times 10^{6}, 14.9 \times 10^{6}$, $17.6 \times 10^{6} \mathrm{~cm} / \mathrm{s}$, and $367,474,607,712 \mathrm{~K}$. The solid squares correspond to the fitting $\omega^{2}=\omega^{2}\left(T_{e}\right)-\left(v_{x} q \cos \varphi\right)^{2}$ for different electric fields.

$\omega=\omega_{p}^{\prime}+\mathbf{v}_{x} \cdot \mathbf{q}=\omega_{p}^{\prime}+v_{x} q \cos \varphi$, with $\omega_{p}^{\prime}$ being the plasmon frequency in the absence of the driving electric field. Thus, the plasmon frequency in a conventional 2DEG depends very strongly on $F_{x}$ via electron drift velocity $v_{x}$ and, therefore, on the angle $\varphi$. This implies the following features. (i) The stronger dependence of the plasmon frequency on $\varphi$ can be observed at a larger electric field, similar to that in graphene. (ii) The maximum plasmon frequency appears at $\varphi=0^{\circ}$ and the minimum is at $\varphi=180^{\circ}$, different from the case of graphene. (iii) The plasmon frequency increases with driving electric field when $\varphi=\left(0^{\circ}, 90^{\circ}\right)$, whereas it decreases with increasing $F_{x}$ when $\varphi=\left(90^{\circ}, 180^{\circ}\right)$, in sharp contrast to that in graphene. There are two major reasons why the plasmon frequency in graphene depends rather weakly on the angle $\varphi$. First, the angular dependence of the plasmon frequency is determined by the energy and momentum conservation laws during the electronic transition events. The Fermi velocity of electrons in graphene is normally much bigger than the drift velocity of electrons so that $v_{x} / v_{F} \ll 1$. Thus, $v_{x}$ has a weaker effect on plasmon frequency, as shown by Eqs. (4) and (5). As a result, the plasmon frequency depends weakly on $\varphi$ in graphene. Second, the electrons in graphene have a linear energy spectrum, which differs significantly from the parabolic energy spectrum for electrons in a conventional 2DEG. Practically, it is difficult to get the analytical expression for the plasmon modes in graphene in the presence of driving electric field, even under the long-wavelength approximation. However, as shown in Fig. 1, we find that the plasmon frequency in graphene in the presence of a driving electric field can be fitted by a formula

$$
\omega^{2}=\omega^{2}\left(T_{e}\right)-\left(v_{x} q \cos \varphi\right)^{2},
$$

which is a functional form of electric temperature $T_{e}$ and electric velocity $v_{x}$ determined simultaneously at a given electric field, with

$$
\omega^{2}\left(T_{e}\right)=\omega_{p}^{2} \int_{0}^{\infty} \frac{d x}{e^{\left(x \cdot E_{F}-\mu\right) / k_{B} T_{e}}+1} .
$$




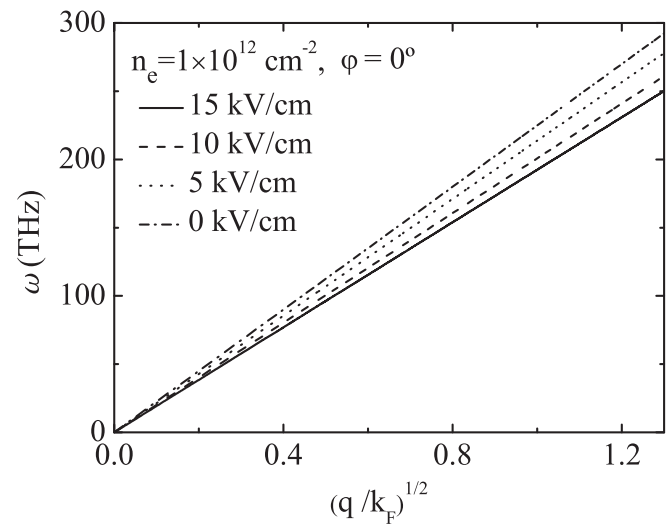

FIG. 2. The dispersion relation of the plasmon modes for different electric fields at the fixed electron density and angle $\varphi$, with $k_{F} \simeq$ $1.77 \times 10^{6} \mathrm{~cm}^{-1}$. The corresponding electron velocities and electron temperatures are given in Fig. 1.

In the absence of the electric field (i.e., $v_{x}=0$ and $T_{e}=T$ with $T$ the lattice temperature), we have

$$
\omega^{2}(T)=\left(\frac{2 e^{2} q}{\hbar^{2} \kappa}\right)\left[\mu+k_{B} T \ln \left(1+e^{\left(-\mu / k_{B} T\right)}\right)\right],
$$

a well-known result for plasmon frequency in graphene at $T \neq 0$.

In Fig. 2, we show the dispersion relation of the plasmon modes in graphene at the fixed electron density and $\varphi=0^{\circ}$ for different electric fields. As depicted, the plasmon frequency in graphene in the presence of driving electric filed maintains the dependence on $q^{1 / 2}$, similar to the case of $F_{x}=0[12,33]$. We can see that the plasmon frequency decreases with increasing electric field over all $q$ regimes. This is attributed to the drifted distribution of electrons in graphene in the presence of the driving electric field. This can result in a shift of the plasmon frequency by the presence of the electric field. We notice that the plasmon frequency in graphene still ranges from terahertz to infrared bandwidth when the driving electric field is present. This implies that plasmons in graphene can couple strongly with the infrared radiation field $[8,10]$. The results shown in Fig. 1 and Fig. 2 demonstrate that the plasmon modes in graphene can be tuned not only by the gate voltage (which changes mainly the electron density) but also by the driving electric field.

In Fig. 3, the dispersion relation for coupled plasmonphonon modes, $\Omega_{+}$and $\Omega_{-}$, are shown for different driving electric fields. We can see that the plasmons in graphene can strongly couple with optical-phonon modes when their energies match somewhere around $q_{0}$, which is the crossover between the uncoupled plasmon energy and optic-phonon energy (see inset in Fig. 3). As depicted in Fig. 3, when $q<q_{0}$, the coupled plasmon-phonon modes in the lower-frequency branch are plasmonlike. Its frequency depends strongly on $q$. For the higher-frequency branch, the plasmon modes are phononlike, which depends rather weakly on $q$. When $q>q_{0}$, the coupled plasmon-phonon modes in the lower-frequency branch become phononlike and the higher-frequency branch is plasmonlike. We find that the $q_{0}$ value, where the nature of dispersion relation changes, increases with increasing driving

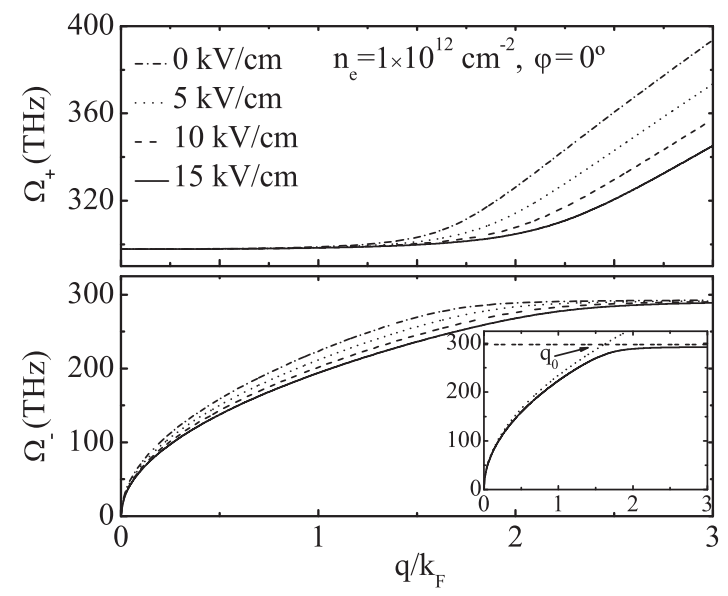

FIG. 3. The dispersion relation for coupled plasmon-phonon modes for different electric fields at $\varphi=0^{\circ}$ and a fixed electron density, with $k_{F} \simeq 1.77 \times 10^{6} \mathrm{~cm}^{-1}$. Inset: the dispersion relation of lower frequency branch of coupled plasmon-phonon frequency (solid), the uncoupled plasmon frequency (dot) and the optical phonon frequency (dash) at electron density $n_{e}=1 \times 10^{12} \mathrm{~cm}^{2}$ and $F_{x}=0$ $\mathrm{kV} / \mathrm{cm}$. Here $q_{0}$ is the crossover between the uncoupled plasmon frequency and optic-phonon frequency. The corresponding electron velocities and electron temperatures are given in Fig. 1.

electric field. At the point $\left(q_{0}, \omega_{q}\right)$ where the the uncoupled plasmon and phonon dispersion across (see, inset in Fig. 3), the strongest coupling can be achieved because the plasmon energy matches the optic-phonon energy. One normally can easily evaluate the strength of the coupling by calculating the frequency difference between the coupled modes $\Omega_{+}$ and $\Omega_{-}$in units of the uncoupled plasmon frequency value $\Delta \Omega / \omega$ at $q_{0}$. Because the plasmon frequency decreases with increasing driving electric filed as indicated by Fig. 2, $q_{0}$ increases with $F_{x}$. Thus, the plasmon modes couple with optic phonons at longer wave vectors with increasing $F_{x}$. Moreover, the frequencies of coupled plasmon-phonon modes in two branches decrease with increasing driving electric field. We can see that the higher-frequency branch depends more strongly on $F_{x}$. These findings indicate that the driving electric field can affect rather strongly the interactions between plasmons and optic phonons in graphene.

Figure 4 shows the angular dependence of the highfrequency branch (upper panel for $q=2.5 k_{F}>q_{0}$ ) and lowfrequency branch (lower panel for $q=k_{F}<q_{0}$ ) of coupled plasmon-phonon modes in graphene for different driving electric fields at a fixed electron density. We can see that the angular dependence of the plasmonlike high-frequency branch when $q>q_{0}$ and low-frequency branch as $q<q_{0}$ for coupled plasmon-phonon modes is similar to the plasmon modes depicted in Fig. 1. For the phononlike high-frequency branch when $q<q_{0}$ and low-frequency branch as $q>q_{0}$, the coupled plasmon-phonon frequency depends weakly on the driving electric field, similar to those shown in Fig. 3. We find that these phononlike modes show a very weak angular dependence in the presence of $F_{x}$.

It has been observed experimentally [24] that, in sharp contrast to conventional semiconductor-based electronic devices, the current saturation is often incomplete in graphene 


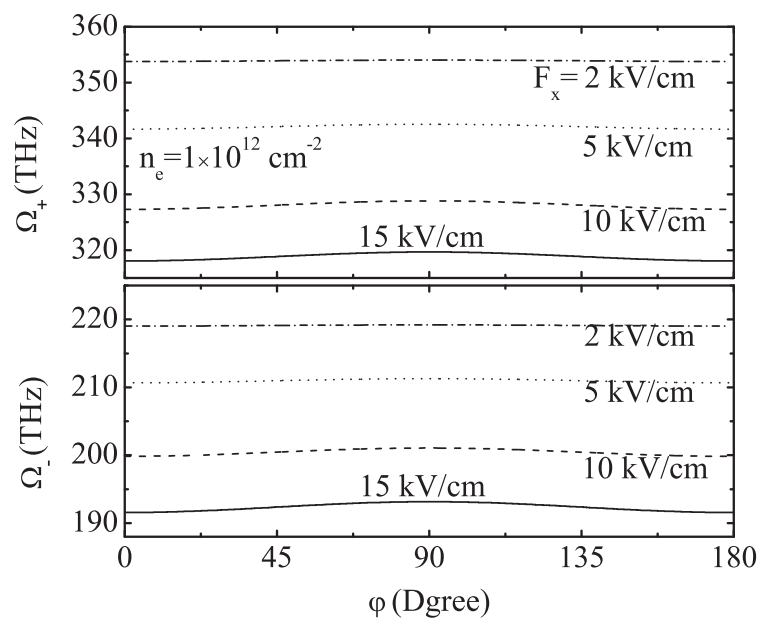

FIG. 4. The angular dependence of the high-frequency branch (upper panel for $q=2.5 k_{F}>q_{0}$ ) and the low-frequency branch (lower panel for $q=k_{F}<q_{0}$ ) of coupled plasmon-phonon modes in graphene for different driving electric fields at a fixed electron density. The corresponding electron velocities and electron temperatures are given in Fig. 1.

samples even at very high $V_{s d}$ voltages. For example, the current can still be well measured for a graphene sample when $V_{s d}>2 \mathrm{~V}$ [24]. The electric field strength $F_{x}$ used in the present calculation is within the touch of the experimental measurements. For example, $F_{x}=15 \mathrm{kV} / \mathrm{cm}$ corresponds to $V_{s d}=1.95 \mathrm{~V}$ at which the current can be measured experimentally for graphene devices [24]. Moreover, because the experimental measurement can be conducted in vacuum [24], the effect of the polarization or ionization of air by strong dc electric field can be avoided. Hence, it is possible to observe experimentally the influence of the driving electric field on plasmon and coupled plasmon-phonon modes in graphene.

\section{CONCLUSIONS}

In this work, we have theoretically investigated the plasmon and coupled plasmon-phonon modes induced by intraband electron-electron interaction in graphene in the presence of driving electric field at room temperature. The main conclusions that we have obtained are as follows.

The frequencies of plasmon and coupled plasmon-phonon modes in graphene depend differently on driving electric field from those in conventional 2DEG systems with a parabolic energy spectrum. Because the Fermi velocity of electrons in graphene is normally much larger than the drift velocity of electrons, the plasmon and coupled plasmon-phonon modes in graphene show a weaker angular dependence than that in conventional 2 DEG systems. We have found that the plasmon and coupled plasmon-phonon frequencies in graphene decrease with increasing driving electric field at room temperature over all angular and wave-vector regimes. Thus, the plasmon and coupled plasmon-phonon modes in graphene can be tuned not only by the gate voltage but also by the driving electric field. These interesting features allow graphene to be more conveniently applied as an advanced plasmonic material. Furthermore, it should be noted that the application of a driving electric field on graphene is electrically equivalent to the injection of electrons into the system. This can result in the generation of plasmon emission. Therefore, graphene can be applied for both plasmonic detection and generation. We hope these important theoretical findings can be verified experimentally.

\section{ACKNOWLEDGMENTS}

This work was supported by the Ministry of Science and Technology of China (Grant No. 2011YQ130018), the Department of Science and Technology of Yunnan Province, the Chinese Academy of Sciences, and by the National Natural Science Foundation of China (Grant No. 11247002).
[1] J. A. Schuller, E. S. Barnard, W. S. Cai, Y. C. Jun, J. S. White, and M. L. Brongersma, Nature Mater. 9, 193 (2010).

[2] N. Yu, Q. J. Wang, M. A. Kats, J. A. Fan, F. Capasso, S. P. Khanna, L. Li, A. G. Davies, and E. H. Linfield, Electronics Lett. 46, S52 (2010).

[3] A. Polman, Science 322, 868 (2008).

[4] D. E. Chang, A. S. Sørensen, P. R. Hemmer, M. D. Lukin, Phys. Rev. Lett. 97, 053002 (2006).

[5] H. A. Atwater, Sci. Am. 17, 56 (2007).

[6] P. R. West, S. Ishii, G. V. Naik, N. K. Emani, V. M. Shalaev, and A. Boltasseva, Laser Photonics Rev. 4, 795 (2010).

[7] M. Jablan, H. Buljan, and M. Soljačić, Phys. Rev. B 80, 245435 (2009).

[8] K. H. L. Frank, D. E. Chang, F. J. Garciade Abajo, Nano Lett. 11, 3370 (2011).

[9] J. N. Chen, M. Badioli, P. Alonso-González, S. Thongrattanasiri, F. Huth, J. Osmond, M. Spasenović, A. Centeno, A. Pesquera, P. Godignon, A. Z. Elorza, N. Camara, F. J. García de Abajo, R. Hillenbrand, and Frank H. L. Koppens, Nature (London) 487, 77 (2012).
[10] Z. Fei, A. S. Rodin. G. O. Andreev, W. Bao, A. S. McLeod, M. Wagner, L. M. Zhang, Z. Zhao, M. Thiemens, G. Dominguez, M. M. Fogler, A. H. Castro Neto, C. N. Lau, F. Keilmann, and D. N. Basov, Nature (London) 487, 82 (2012).

[11] L. Ju, B. S. Geng, J. Horng, C. Girit, M. Martin, Z. Hao, H. A. Bechtel, X. G. Liang, A. Zettl, Y. R. Shen, and F. Wang, Nat. Nanotechnol. 6, 630 (2011).

[12] E. H. Hwang and S. Das Sarma, Phys. Rev. B 75, 205418 (2007).

[13] F. Rana, IEEE Transactions on Nanotechnology 7, 91 (2008).

[14] W. L. Gao, G. Shi, Z. H. Jin, J. Shu, Q. Zhang, R. Vajtai, P. M. Ajayan, J. Kono, and Q. F. Xu, Nano Lett. 13, 3698 (2013).

[15] Q. L. Bao and K. P. Loh, ACS Nano. 6, 3677 (2012).

[16] A. S. Bhatti, D. Richards, and H. P. Hughes, D. A. Ritchie, J. E. F. Frost, and G. A. C. Jones, Phys. Rev. B 51, 2252 (1995).

[17] K. Kempa, Appl. Phys. Lett. 50, 1185 (1987).

[18] P. Bakshi, J. Chen, and K. Kempa, J. Appl. Phys. 64, 2243 (1988).

[19] H. M. Dong, L. L. Li, W. Y. Wang, S. H. Zhang, C. X. Zhao, and W. Xu, Physica E 44, 1889 (2012).

[20] T. Ando, J. Phys. Soc. Jpn. 76, 024712 (2007). 
[21] W. Xu, F. M. Peeters, and J. T. Devreese, Phys. Rev. B 43, 14134 (1991).

[22] H. M. Dong, W. Xu, and F. M. Peeters, J. Appl. Phys. 110, 063704 (2011).

[23] W. Xu and C. Zhang, Phys. Rev. B 55, 5259 (1997).

[24] A. Barreiro, M. Lazzeri, J. Moser, F. Mauri, and A. Bachtold, Phys. Rev. Lett. 103, 076601 (2009).

[25] H. M. Dong, W. Xu, Z. Zeng, T. C. Lu, and F. M. Peeters, Phys. Rev. B 77, 235402 (2008).

[26] M. Jablan, M. Soljačić, and H. Buljan, Phys. Rev. B 89, 085415 (2014).
[27] G. W. Hanson, E. Forati, W. Linz, and A. B. Yakovlev, Phys. Rev. B 86, 235440 (2012)

[28] W. Xu, F. M. Peeters, and T. C. Lu, Phys. Rev. B 79, 073403 (2009).

[29] S. Das Sarma and E. H. Hwang, Phys. Rev. B 54, 1936 (1996).

[30] I. Grosu and L. Tugulan, Physica E 40, 474 (2008).

[31] G. F. Giuliani and J. J. Quinn, Phys. Rev. B 29, 2321 (1984).

[32] C. X. Zhao, W. Xu, and F. M. Peeters, Appl. Phys. Lett. 102, 222101 (2013).

[33] V. Ryzhii, A. Satou, and T. Otsuji, J. Appl. Phys. 101, 024509 (2007). 